\title{
A Novel Design of CPW Feed Planar Omnidirectional Circularly Polarized Antenna
}

\author{
Jiawei Yang, ${ }^{1} \mathrm{Xi} \mathrm{Li}\left(\mathbb{D},{ }^{2}\right.$ and Lin Yang ${ }^{2}$ \\ ${ }^{1}$ Guangdong Mikwave Communication Tech, Xidian University, Guangzhou, Guangdong 510700, China \\ ${ }^{2}$ Xidian University, Xi'an, Shannxi 710071, China \\ Correspondence should be addressed to Xi Li; xli@xidian.edu.cn
}

Received 15 December 2020; Revised 7 February 2021; Accepted 17 March 2021; Published 28 March 2021

Academic Editor: Mauro Parise

Copyright (c) 2021 Jiawei Yang et al. This is an open access article distributed under the Creative Commons Attribution License, which permits unrestricted use, distribution, and reproduction in any medium, provided the original work is properly cited.

A novel planar omnidirectional circularly polarized (CP) antenna is presented. The omnidirectional circular polarization characteristics of the planar antenna are the result of the combined effect of a planar quasi-magnetic dipole (PQMD) and a printed electric dipole (PED) in this paper. The CP radiation pattern of the proposed antenna can be achieved by distributing appropriate current amplitude and phase to both elements, respectively. A power divider is used to adjust the amplitude and phase relationship between two basic components. In order to achieve a planar structure, coplanar waveguide (CPW) feed is adopted to feed the quasi-magnetic dipole for the first time. The overall electrical size of antenna is $1.61 \lambda \times 0.38 \lambda$. Finally, the correctness of our theoretical analysis is verified by processing and measuring this antenna. Through the analysis of the measurement results, we have obtained the following conclusions: the operating bandwidth of the proposed antenna obtained by measurement is from 5.67 to $5.86 \mathrm{GHz}$, in which the reflection coefficient is less than $-10 \mathrm{~dB}$ and the axial ratio is less than $3 \mathrm{~dB}$. Within the available bandwidth, the proposed antenna achieves omnidirectional radiation characteristics with a gain between 0.89 and $2.48 \mathrm{dBic}$.

\section{Introduction}

Omnidirectional $\mathrm{CP}$ antennas have got more and more attention because such an antenna has $\mathrm{CP}$ property and produces omnidirectional radiation pattern. These characteristics can reduce the effect of Faraday rotation and multipath interference on antenna transmission [1]. Besides, the omnidirectional radiation pattern can make one antenna cover a larger area and optimize the system configuration. Hence, a large number of omnidirectional $\mathrm{CP}$ antennas are applied to the wireless communication systems [2-4].

More and more ways have been put forward to study and design omnidirectional CP antennas over the last few decades [5-8]. The Lindenblad antenna is considered to be the first omnidirectional CP antenna. Up to now, the antenna is still widely used in various fields. Additionally, an outstanding CP antenna has been presented to obtain an ideal omnidirectional pattern [9]. In recent years, various types of antennas have researched on the omnidirectional CP characteristics.
The omnidirectional $\mathrm{CP}$ antenna in the form of dielectric resonator is reported in [8-10].

Most recently, a new omnidirectional CP antenna array is designed by connecting four $\mathrm{CP}$ antenna elements and one parallel strip-line feeding network in [11]. Nevertheless, studies [5-11] do not achieve the omnidirectional CP antenna using a planar structure. In contrast to this antenna, in [12], a planar CP antenna with a planar structure and a smaller size using magnetic-electric dipole principle was presented. However, its disadvantage is still obvious. Improper feeding position can cause inconvenient use and deterioration of the performance. Besides, a low-profile omnidirectional CP antenna with planar sector structure is reported in [13]. In addition to complex structure, this antenna has a bigger ground and it cannot be integrated with printed circuit board.

In this paper, a novel planar omnidirectional $\mathrm{CP}$ antenna is presented. The omnidirectional CP antenna is composed of a PQMD, a PED, and a power divider. Due to the introduction of the power divider, it is more convenient to 
control the amplitude and phase of the two elements, wherein one port of the power divider is connected to the printed electric dipole and the other port is connected to the quasi-magnetic dipole feeding in the form of CPW. Such a configuration can easily produce an omnidirectional $\mathrm{CP}$ radiation pattern. Its advantages are as follows. In the vertical plane, the antenna realizes a planar physical structure. Besides, the feed point at the bottom of the antenna does not affect the characteristic of the antenna. In terms of processing, the antenna is easily machined and easy to integrate with the printed circuit board (PCB). In the following sections, we will give a theoretical analysis and equivalent model of the antenna. Meanwhile, the detailed process of designing and optimizing the antenna is also given. Finally, the comparative analysis is presented at the end.

\section{Theoretical Analyses and Antenna Design}

2.1. Theoretical Analyses. As shown in Figure 1, the prerequisite for the generation of $\mathrm{CP}$ waves by the proposed antenna is to generate two orthogonal waves with 90 -degree phase difference. For orthogonal waves, the decisive factor is to keep the equal magnitude condition of $E_{\theta}$ and $E_{\Phi}$ in the azimuth plane. As studied in [14], the equivalent source formulation is presented in Figure 1. The proposed antenna is analyzed by combining a PQMD and a PED. The magnetic current at the radiation plane of the rectangular patch can be known as $M_{\mathrm{z}}=\mathrm{A} \delta(z)$ (A is known as the magnitude of the magnetic current). The electric current on the microstrip line can be approximately assumed as $J_{\mathrm{z}}=\mathrm{B} \delta(z)$ (B is assumed as the magnitude of the electric current).

It can be concluded that the field component $E_{\theta}$ is contributed by the magnetic current, whereas the field component $E_{\Phi}$ is produced by the electric current. Actually, the generation of the field is coincident with the study in [13], and the far-field radiation pattern generated by the PED is expressed as

$$
E_{\theta}=-j k_{0} \frac{e^{-j k_{0} r}}{4 \pi r}\left(\eta_{0} f_{x} \cos \theta \cos \varphi-f_{m x} \sin \varphi\right) .
$$

Similarly, the quasi-magnetic dipole placed along the $z$ axis produces radiation pattern by the aperture of the rectangular patch; its two planes are placed on both sides of the medium. The far-field radiation pattern generated by the PQMD is expressed as

$$
E_{\varphi}=j k_{0} \frac{e^{-j k_{0} r}}{4 \pi r}\left(\eta_{0} f_{x} \sin \varphi+f_{m x} \cos \theta \cos \varphi\right) .
$$

The specific implementation formula for left-hand CP (LHCP) and right-hand CP (RHCP) can be expressed as follows:

$$
\begin{aligned}
& E_{\mathrm{LH}}=\frac{1}{\sqrt{2}}|E \theta-j E \varphi|, \\
& E_{\mathrm{RH}}=\frac{1}{\sqrt{2}}|E \theta+j E \varphi|,
\end{aligned}
$$

and $\mathrm{AR}$ in the xoy plane is determined as

$$
A R(d B)=20\left|\log _{10}\left(\frac{E_{\theta}(\theta)}{E_{\varphi}(\theta)}\right)\right| .
$$

It is seen that AR is mainly controlled by the amplitude and phase of PQMD and PED.

2.2. Physical Structure. The specific structural dimensions are indicated in Figures 2(a) and 2(b). The antenna is manufactured on a substrate with a relative permittivity of $\varepsilon_{\mathrm{r}}$. The outer dimensions of the substrate are Ls $\times \mathrm{Ws} \times h$. A half-wave printed dipole patch is machined on the substrate. The outer dimensions of the dipole patch are $\mathrm{Wd} \times \mathrm{Ld}$. There is an $\mathrm{Lr} \times \mathrm{Wr}$ size rectangular patch located at the bottom of the dipole patch. In order to generate an equivalent electronic wall, nine metal vias are placed at the side of the rectangular patch. A power divider is introduced to realize power distribution. One output port is connected to the printing dipole patch, and the other one is connected to the rectangular patch by CPW feeding mode. The distance between the two ports is a wavelength because of the demand for orthogonal phase. The length is analyzed by software TXTline and defined as Lq. At last, a $50 \Omega$ microstrip line connects the two output ports with the length of Lp. At the bottom of the circuit board, a truncated ground is printed on its back side. Detailed dimensions are given in Table 1.

\section{Parametric Studies}

In this section, in order to achieve a more in-depth understanding of the working principle, we have conducted a series of parameter comparisons to understand the effect of the relevant dimensions on the antenna performance. The parameters of main concern are the length of the printed dipole patch $(\mathrm{Ld})$, the width of the rectangular patch (Lr), and the distance between the rectangular patch and printed dipole patch (Lq). They are chosen because they are important in designing the proposed antenna. Ansoft High-Frequency Structure Simulator software is used to analyze these parameters.

3.1. Printing Dipole Parameter Optimization. According to the theoretical analyses, the length of the dipole patch is about half a wavelength because the dipole patch is connected partly to the substrate with a relative permittivity of $\varepsilon_{\mathrm{r}}$ and partly to the air; we can conclude that the length of the dipole patch can be initially set to a value in the middle of these two sizes. Figure 3 presents the variation of reflection coefficients and AR under three different printed dipole patch lengths.

Both the reflection coefficients and AR are sensitive to the length of Ld. It is observed that the resonant frequency of the proposed antenna is shifted to the low frequency with the increasing size of $\mathrm{Ld}$. As for $\mathrm{AR}$, medium value can get an ideal AR effect. When Ld is equal to 13, the antenna will produce an ideal resonant frequency and have a lowest $A R$ in azimuth plane. 


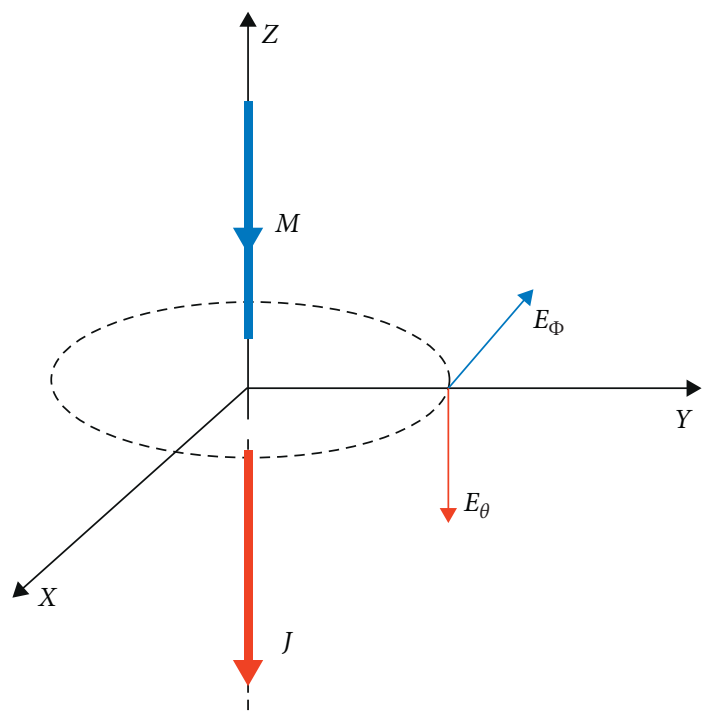

Figure 1: Equivalent model from the view of the proposed antenna.

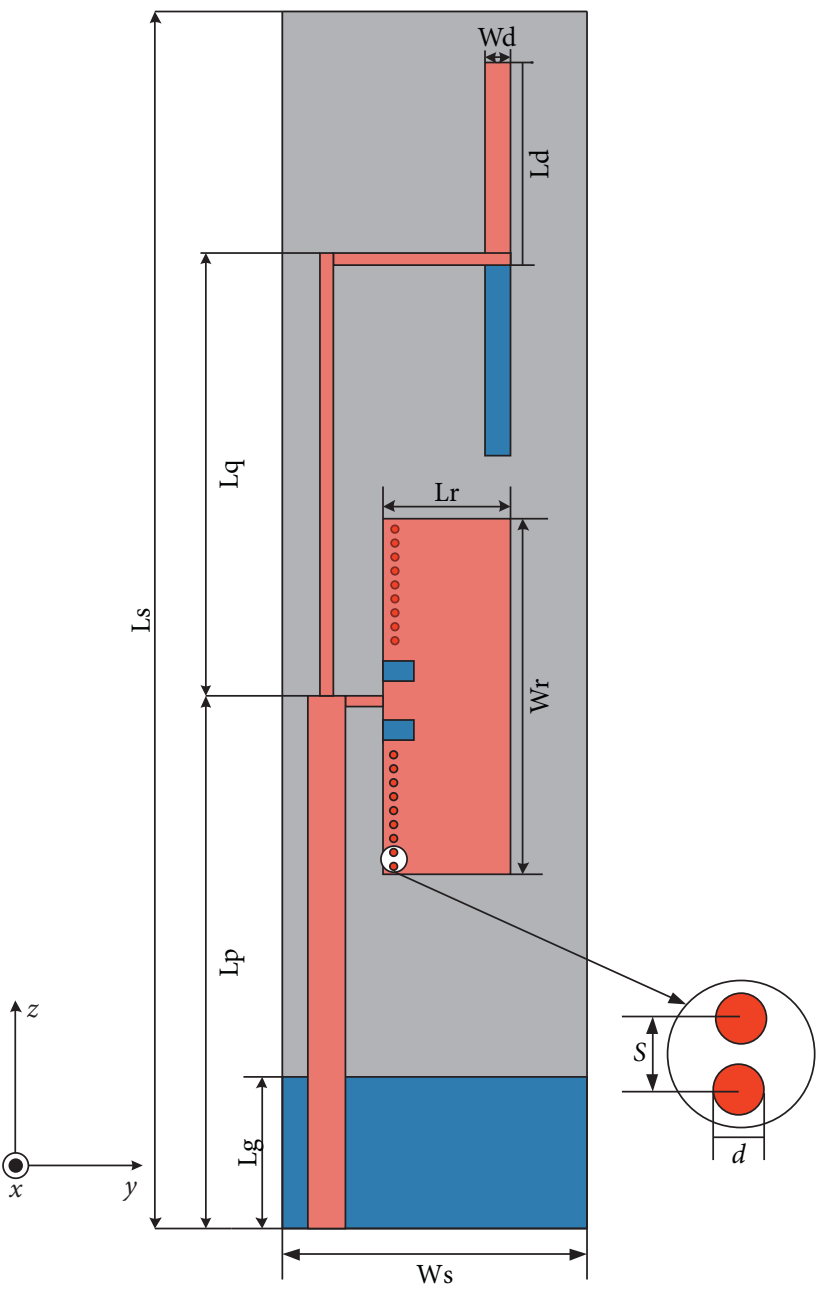

(a)
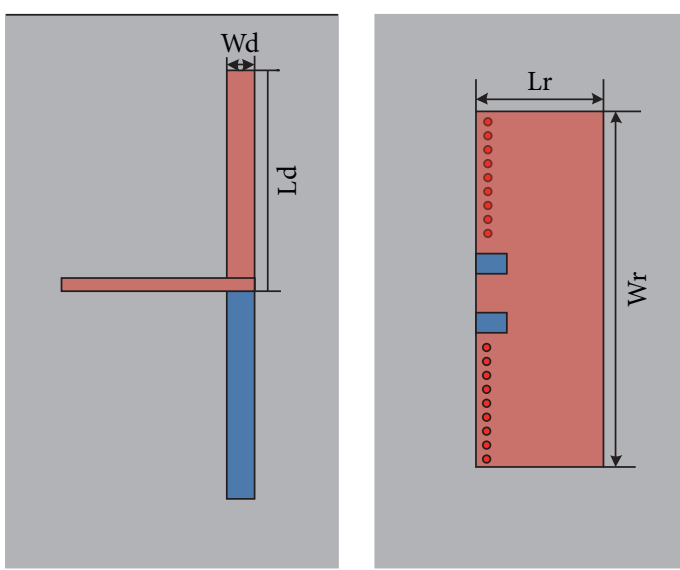

(b)

FIGURE 2: Configuration of the proposed antenna: (a) the top patch and (b) the printed dipole patch and the rectangular patch. 
TABLE 1: Design parameters of the proposed omnidirectional antenna.

\begin{tabular}{lccc}
\hline Parameter & Value & Parameter & Value \\
\hline Ws & $20 \mathrm{~mm}$ & Lq & $34.5 \mathrm{~mm}$ \\
Ls & $84 \mathrm{~mm}$ & Lg & $10 \mathrm{~mm}$ \\
Wd & $2.2 \mathrm{~mm}$ & W1 & $0.73 \mathrm{~mm}$ \\
Ld & $13.35 \mathrm{~mm}$ & $S$ & $1 \mathrm{~mm}$ \\
Wr & $8.26 \mathrm{~mm}$ & $d$ & $0.6 \mathrm{~mm}$ \\
Lr & $31 \mathrm{~mm}$ & $h$ & $1 \mathrm{~mm}$ \\
Lp & $33.62 \mathrm{~mm}$ & $\varepsilon_{\mathrm{r}}$ & 2.65 \\
\hline
\end{tabular}

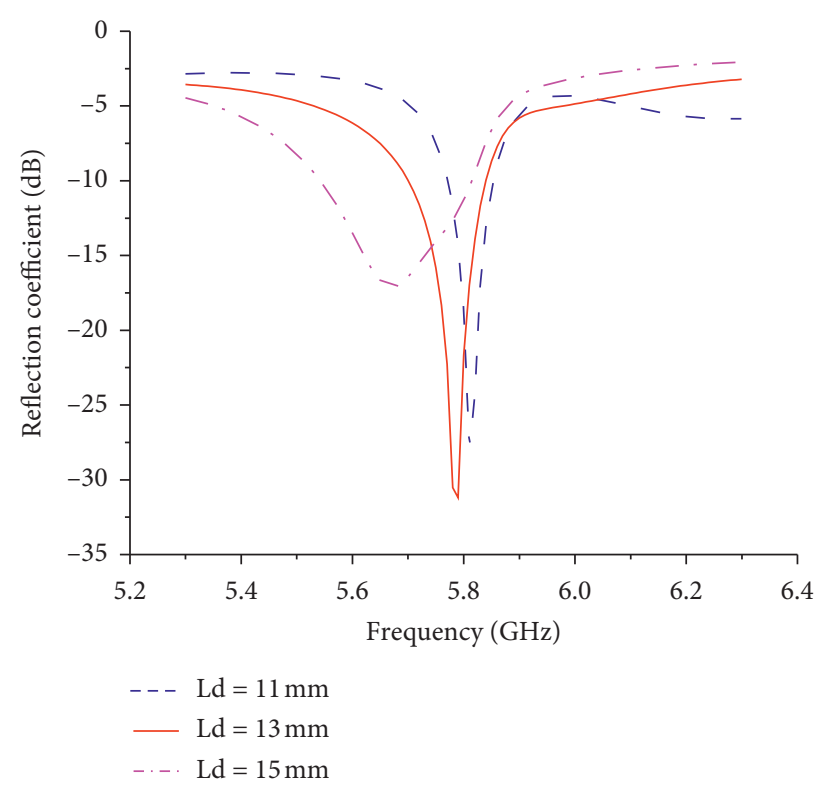

(a)

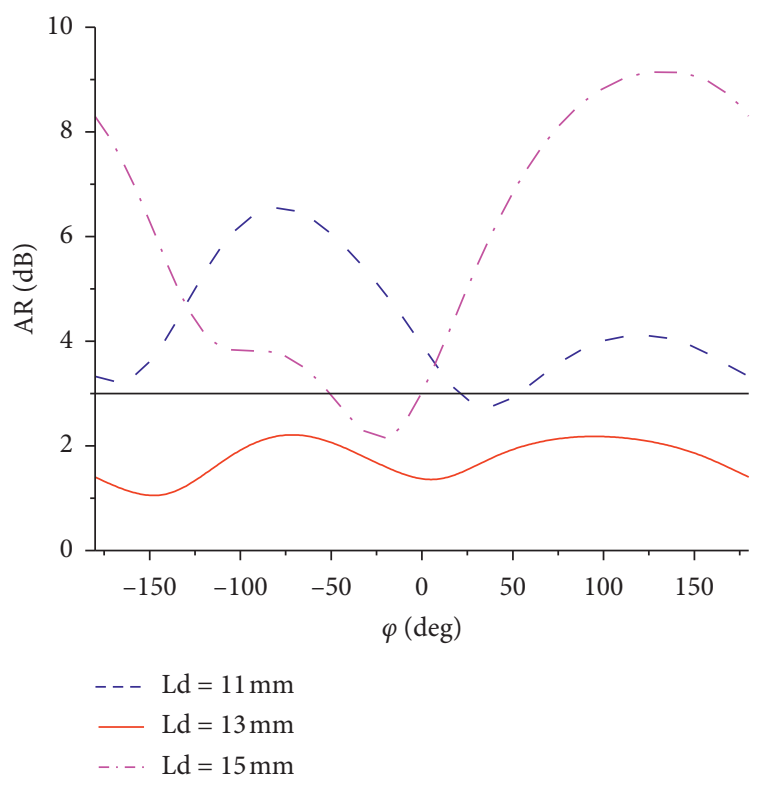

(b)

FIgURE 3: The influence of parameter Ld on antenna performance.

3.2. Rectangular Patch Parameter Optimization. The changes of Lr affects the reflection coefficient and AR that are presented in Figure 4. As can be seen, Ld and Lr have a similar effect on the resonant frequency of the antenna. Compared with the effect of $\mathrm{Ld}$, the width of the rectangular patch $\mathrm{Lr}$ has a bigger influence on AR. It is observed that the resonant frequency appears at $5.8 \mathrm{GHz}$ and $\mathrm{AR}$ is the lowest for $\mathrm{Lr}=8.26 \mathrm{~mm}$. Hence, the value $\mathrm{Lr}=8.26 \mathrm{~mm}$ can be viewed as a final value for the proposed antenna.

3.3. Phase Delay Line. Figure 5 shows the effects of Lq on the reflection coefficient and AR. As predicted, AR will deteriorate as the phase changes. It is preferred to adopt a wavelength phase delay line to ensure the phase relationships between the two elements. Meanwhile, its characteristic impedance should always remain at $100 \mathrm{ohms}$ because the input impedance of PQMD and PED is 100 ohms. In this case, input port can match 50 ohms.

In conclusion, the AR of radiation pattern is mainly controlled by Ld, Lr, and Lq. The critical dimension of the controlling operation frequency is Ld and Lr. When the PED and PQMD are in the resonant case, Lq will be the most important parameter in the designing process by controlling the phase.

\section{Experimental Verification}

The proposed antenna in this paper working at $5.8 \mathrm{GHz}$ is manufactured by utilizing printed circuit board (PCB) technology. Figure 6 shows the physical image of the proposed antenna. Using Agilent's Vector Network Analyzer as a measuring instrument, the measured reflection coefficient is presented in Figure $7(\mathrm{a})$. It can be observed that the measured result is close to the simulated one, while there still exists a slight frequency drift; it may be caused by the dielectric constant offset used in the manufacture and the accuracy of the microstrip line processing dimensions.

It can be seen that AR varies with frequency in Figure 7(b). The measured result presents that the proposed antenna has an AR below $3 \mathrm{~dB}$ in the azimuth plane in the band from $5.67 \mathrm{GHz}$ to $5.87 \mathrm{GHz}$. From the figure, we can also see that AR is not better than the simulation. Fortunately, within the effective bandwidth, we have successfully achieved an AR less than $3 \mathrm{~dB}$ in the azimuth plane.

The far-field patterns in the elevation and azimuth plane at $5.8 \mathrm{GHz}$ are shown in Figures 8 (a) and $8(\mathrm{~b})$. It can be observed that the proposed antenna achieves an omnidirectional LHCP pattern with an out-of-roundness less than $3 \mathrm{~dB}$ in the azimuth plane. The consistency of the 


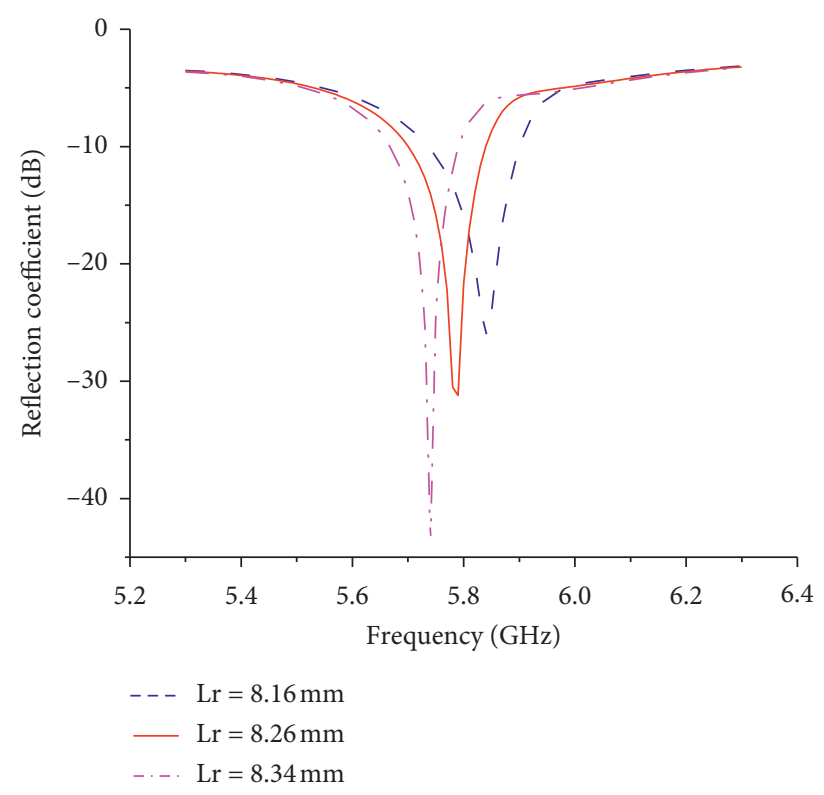

(a)

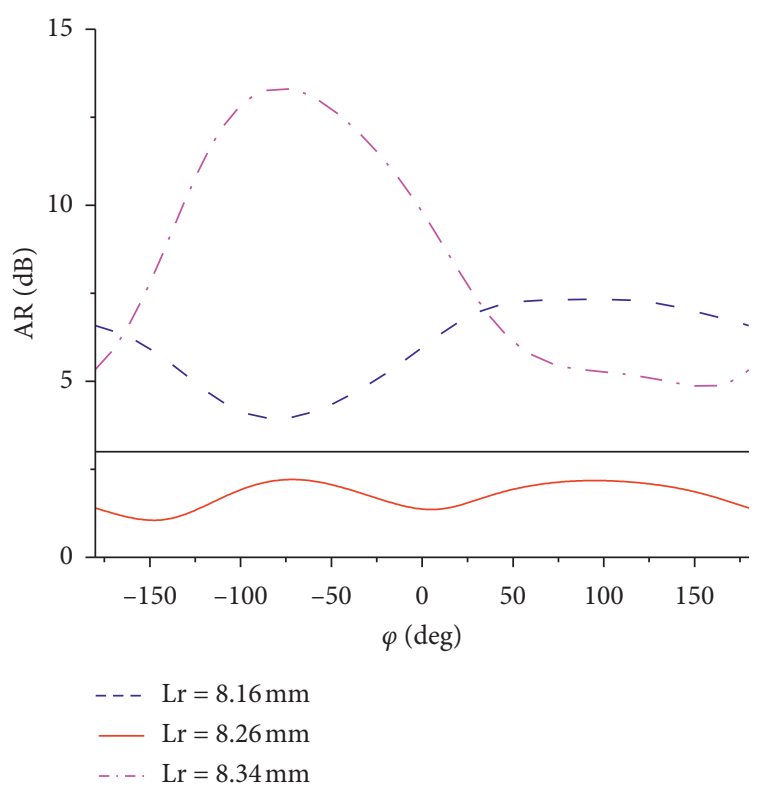

(b)

FIgURE 4: The influence of parameter $\operatorname{Lr}$ on antenna performance.

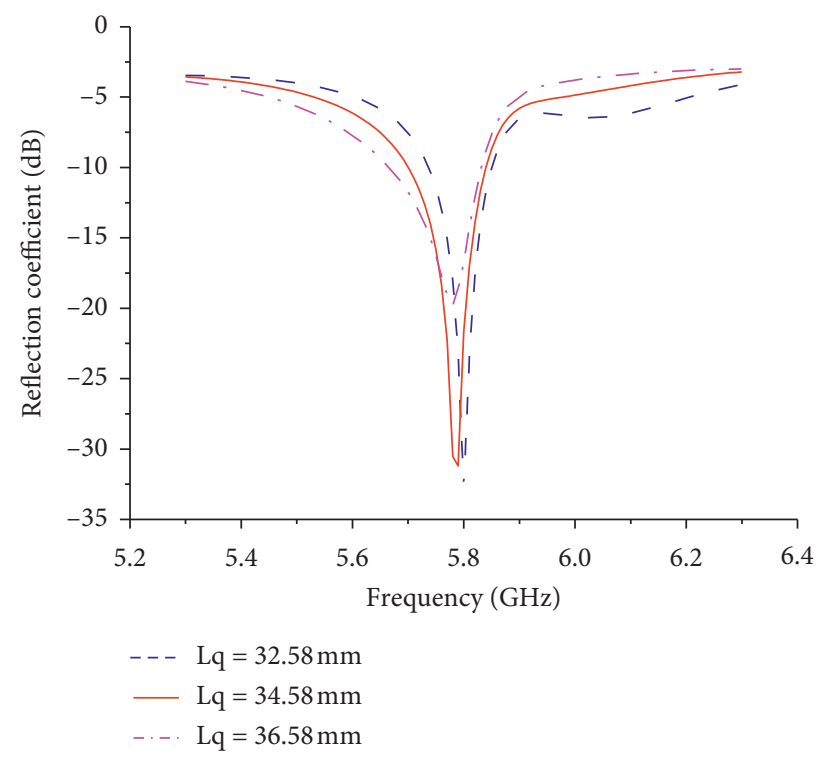

(a)

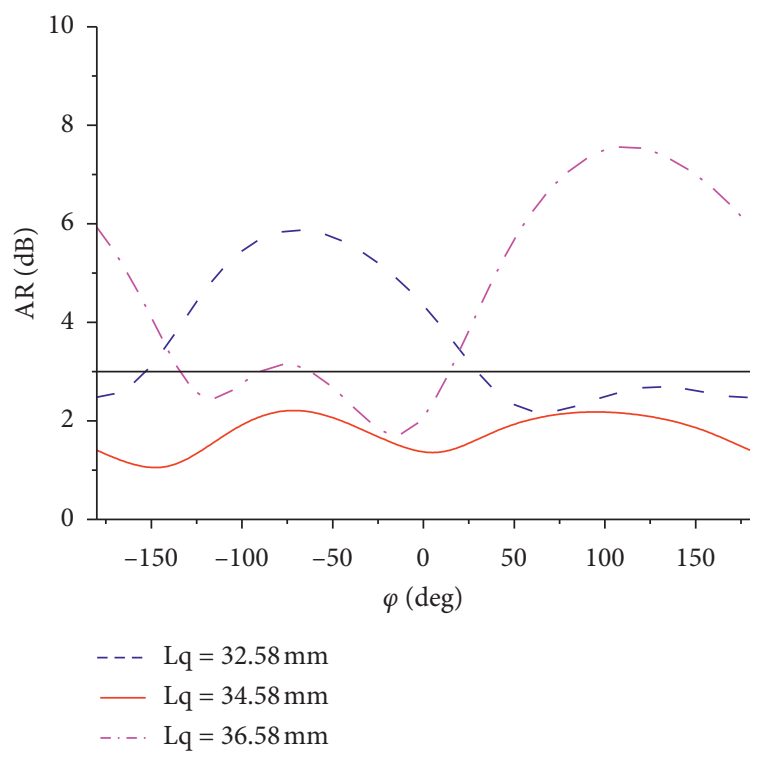

(b)

Figure 5: The influence of parameter Lq on antenna performance.

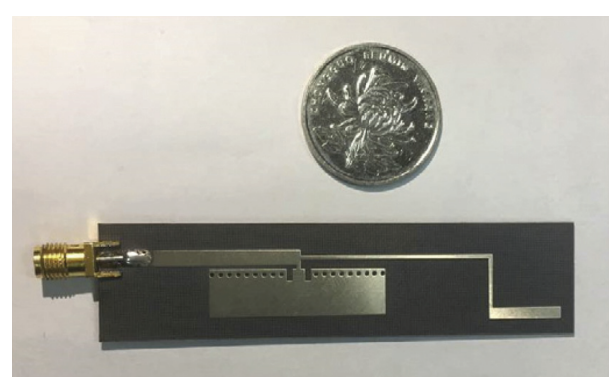

(a)

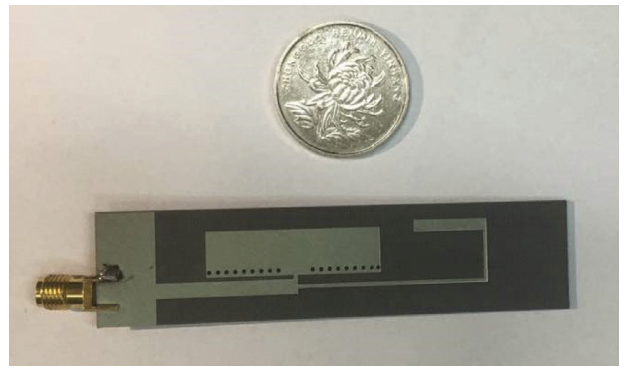

(b)

Figure 6: The fabricated antenna: (a) top view; (b) bottom view. 


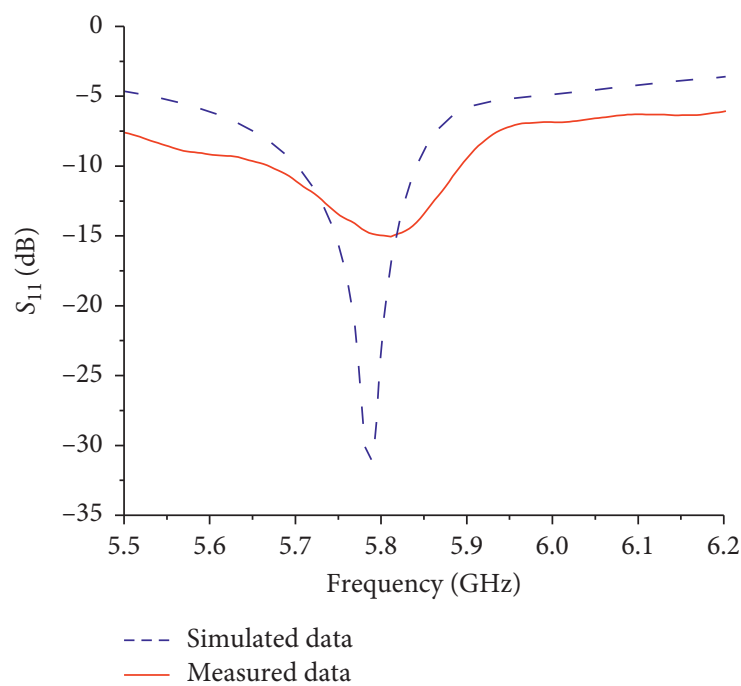

(a)

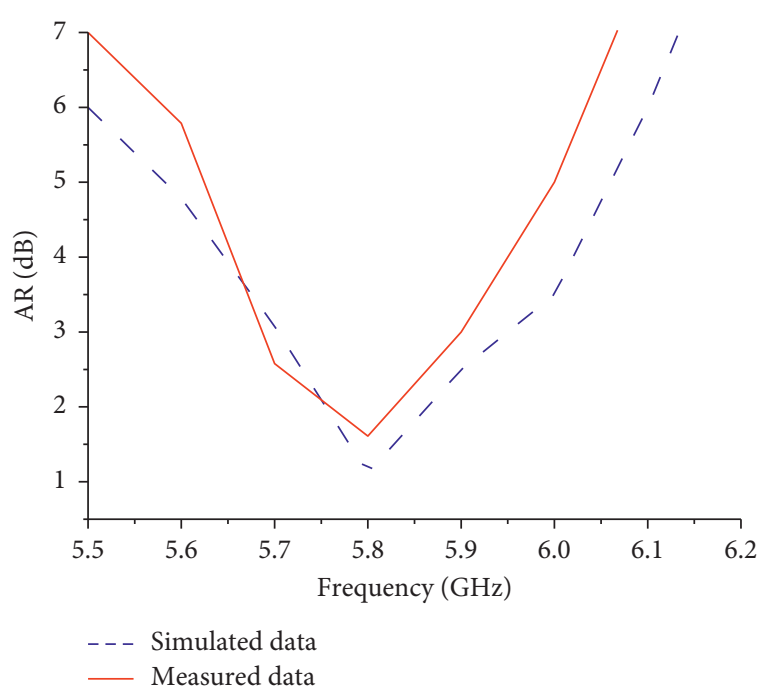

(b)

FIgURE 7: Reflection coefficient and axial ratios.

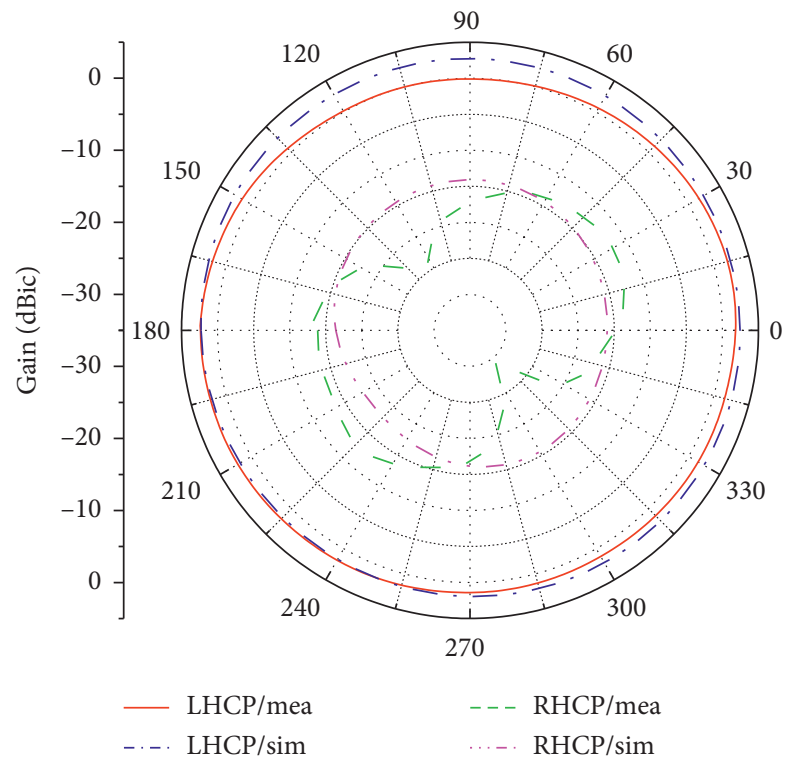

(a)

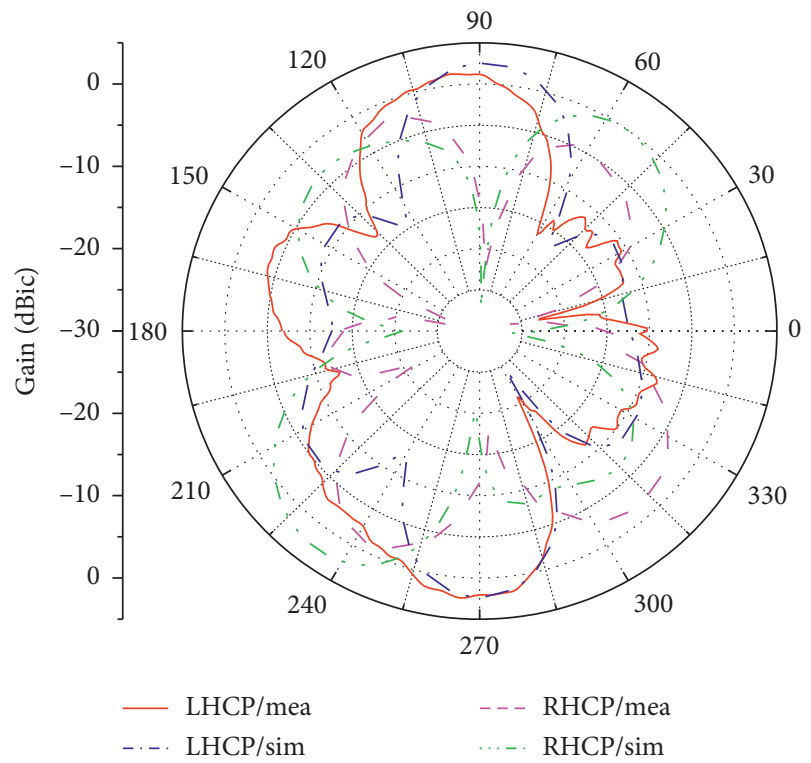

(b)

FIGURE 8: Simulated and measured radiation patterns at $5.8 \mathrm{GHz}$ in the (a) azimuth plane and (b) elevation plane.

measurement results and the simulation results is not strong. The difference between them is mainly due to the unsatisfactory measurement environment. Luckily, within the effective bandwidth, measurement results have reached the design requirements in terms of out-of-roundness and AR.

The maximum gain and radiation efficiency at each frequency point are presented in Figure 9. The proposed antenna has a gain between 0.89 and $2.48 \mathrm{dBic}$ within the entire measurement bandwidth. Except for gain, Figure 9 also presents the radiation efficiency of the proposed antenna. Within the effective bandwidth, the simulated radiation efficiency of the proposed omnidirectional CP antenna is more than $94 \%$. The measured radiation efficiency of the proposed omnidirectional CP antenna is between $92.8 \%$ and $94.7 \%$. From the above expression, we can see that simulated radiation efficiency is higher than the measurement; the reason may be that the simulation software is not accurate for the calculation of conductor loss. 


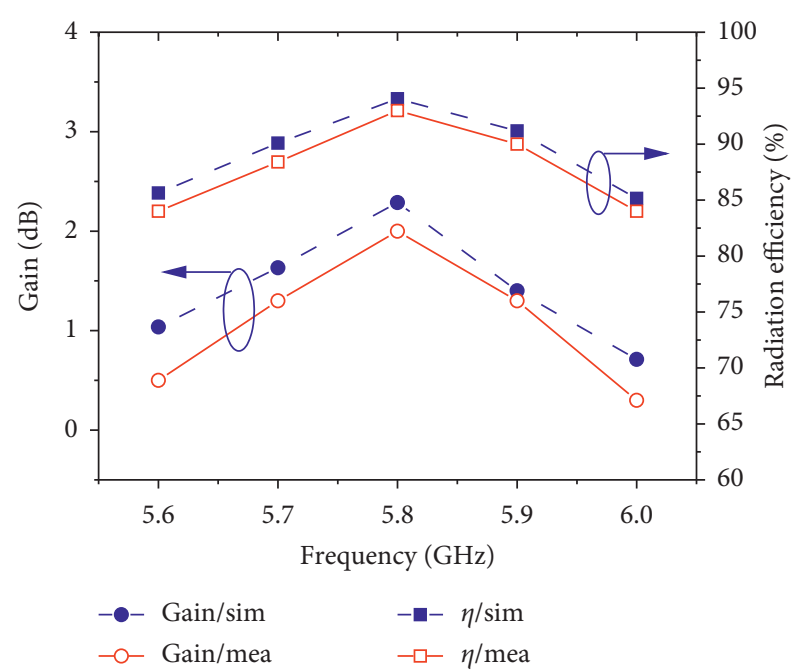

FIGURE 9: Simulated and measured LHCP gain and radiation efficiency.

\section{Conclusion}

In this paper, a novel planar omnidirectional circularly polarized (CP) antenna is presented. The antenna combines a planar quasi-magnetic dipole and a printed electric dipole, and their connection is achieved by power divider. It is worth mentioning that the CPW feed is adopted to feed the quasimagnetic dipole for the first time so as to achieve a planar structure. The proposed antenna can be easily integrated with the PCB and can form an array by using the CPW feed technology. The radiation principle of the antenna can be considered as producing a magnetic current and an electric current. The CP radiation pattern is formed by adjusting the proper amplitude and phase of the electric current and magnetic current. The proposed antenna was designed, processed, and tested. Measured impedance bandwidth of the proposed antenna is $3.3 \%(5.67-5.86 \mathrm{GHz})$. Within the entire measurement bandwidth, the omnidirectional $\mathrm{CP}$ radiation pattern is achieved and the proposed antenna meets design requirements. The proposed antenna can be a good candidate for WLAN application.

\section{Data Availability}

The data used to support the findings of this study are included within the article.

\section{Conflicts of Interest}

The authors declare that they have no conflicts of interest.

\section{References}

[1] S. Gao, Q. Luo, and F. Zhu, Circularly Polarized Antennas, Wiley, Chechester, U.K., 2014.

[2] K. Sakaguchi and N. Hasebe, "A circularly polarized omnidirectional antenna," in Proceedings of the 8th Asia-Pacific Conference on Antennas and Propagation, pp. 477-480, Incheon, Korea, April 1993.
[3] V. Galindo and K. Green, "A near-isotropic circulary polarized antenna for space vehicles," IEEE Transactions on Antennas and Propagation, vol. 13, no. 6, pp. 872-877, 1965.

[4] K. L. Wong, F. R. Hsiao, and C. L. Tang, "A low-profile omnidirectional circularly antenna for WLAN access point," in Proceedings of the IEEE International Symposium on Antennas and Propagation, vol. 3, pp. 2580-2583, Atlanta, GA, USA, June 2004.

[5] B.-C. Park and J.-H. Lee, "Omnidirectional circularly polarized antenna utilizing zeroth-order resonance of epsilon negative transmission line," IEEE Transactions on Antennas and Propagation, vol. 59, no. 7, pp. 2717-2721, 2011.

[6] W. Lin and H. Wong, "Circularly polarized conical-beam antenna with wide bandwidth and low profile," IEEE Transactions on Antennas and Propagation, vol. 62, no. 12, pp. 5974-5982, 2014.

[7] J. Liu, Q. Xue, H. Wong, H. W. Lai, and Y. Long, "Design and analysis of a low-profile and broadband microstrip monopolar patch antenna," IEEE Transactions on Antennas and Propagation, vol. 61, no. 1, pp. 11-18, 2013.

[8] Y. M. Pan, K. W. Leung, and K. Lu, "Omnidirectional linearly and circularly polarized rectangular dielectric resonator antennas," IEEE Transactions on Antennas and Propagation, vol. 60, no. 2, pp. 751-759, 2012.

[9] A. Narbudowicz, X. L. Bao, and M. J. Ammann, "Omnidirectional circularly-polarised microstrip patch antenna," Electronics Letters, vol. 48, no. 11, pp. 614-615, 2012.

[10] Y. M. Pan and K. W. Leung, "Wideband omnidirectional circularly polarized dielectric resonator antenna with parasitic strips," IEEE Transactions on Antennas and Propagation, vol. 60, no. 6, pp. 2992-2997, 2012.

[11] J. Shi, X. Wu, X. Qing, and Z. N. Chen, "An omnidirectional circularly polarized antenna array," IEEE Transactions on Antennas and Propagation, vol. 64, no. 2, pp. 574-581, 2016.

[12] Q. X. Chu, M. Ye, and X. R. Li, “A low-profile omnidirectional circularly Polarized Antenna using plannar sector-shaped endfire elements," IEEE Transactions on Antennas and Propagation, vol. 64, no. 2, pp. 1611-1694, 2017.

[13] J. H. Liu, Y. X. Li, and Z. X. Liang, "A planar quasi-magneticelectric circularly polarized antenna," IEEE Transactions on Antennas and Propagation, vol. 64, no. 2, pp. 2108-2114, 2016.

[14] Y. Shi and J. Liu, "Wideband and low-profile omnidirectional circularly polarized antenna with slits and shorting-vias," IEEE Antennas and Wireless Propagation Letters, vol. 15, no. 2, pp. 686-689, 2016. 